\title{
FINITE GROUPS WHOSE SUBNORMAL SUBGROUPS PERMUTE WITH ALL SYLOW SUBGROUPS
}

\author{
RAM K. AGRAWAL 1
}

ABSTRACT. As a generalization of $(t)$-groups and of $(q)$-groups, a group $G$ is called a $(\pi-q)$-group if every subnormal subgroup of $G$ permutes with all Sylow subgroups of $G$. It is shown that if $G$ is a finite solvable $(\pi-q)$-group, then its hypercommutator subgroup $D(G)$ is a Hall subgroup of odd order and every subgroup of $D(G)$ is normal in $G$; conversely, if a group $G$ has a normal Hall subgroup $N$ such that $G / N$ is a $(\pi-q)$-group and every subnormal subgroup of $N$ is normal in $G$, then $G$ is a $(\pi-q)$-group.

Let $G$ be a finite group. Following Ore [6], a subgroup of $G$ is called quasinormal in $G$ if it permutes with all subgroups of $G$. As a generalization of this concept we say, following Kegel, that a subgroup of $G$ is $\pi$-quasinormal in $G$ if it permutes with all Sylow subgroups of $G$. Kegel [5] proved that a $\pi$-quasinormal subgroup is always a subnormal subgroup. Since a subnormal subgroup is not necessarily a $\pi$-quasinormal subgroup, it seems natural to ask: "What can be said about the structure of a group if all of its subnormal subgroups are $\pi$-quasinormal in the group?" We call such a group a $(\pi-q)$-group and, in this paper, we study finite $(\pi-q)$ groups. We especially study and characterize, in $\$ 2$, finite solvable $(\pi-q)$-groups. Theorems $2.3,2.4$, and 2.5 establish the characterization.

The $(q)$-groups and the $(t)$-groups (see the definitions below) have been studied by Zacher [8] and Gaschütz [2], respectively, and they have characterized such finite solvable groups. We extend their results to finite solvable $(\pi-q)$-groups and give the conditions under which a $(\pi-q)$-group is either a $(q)$-group or a $(t)$-group.

Throughout the paper, the groups are finite.

Received by the editors June 26, 1973 and, in revised form, January 11, 1974. AMS (MOS) subject classifications (1970). Primary 20D10, $20 \mathrm{D} 35$.

Key words and phrases. $\pi$-quasinormal subgroup, $(\pi-q)$-group.

1 The author is indebted to the referee for helpful suggestions. 
1. Definitions and preliminary results.

Definitions. Subgroups $H$ and $K$ of the group $G$ permute if $H K=K H$. A subgroup of $G$ is $\pi$-quasinormal (quasinormal) in $G$ if it permutes with all Sylow subgroups (all subgroups) of $G$. A group $G$ is a $(\pi-q)$-group $((q)$ group) if every subnormal subgroup of $G$ is $\pi$-quasinormal (quasinormal) in $G$, and $G$ is a $(t)$-group if all subnormal subgroups of $G$ are normal in $G$.

We now mention two basic results on $\pi$-quasinormal subgroups which are needed later.

(1.1) [5] If $H \leq K \leq G$ and $H$ is $\pi$-quasinormal in $G$, then $H$ is $\pi$-quasinormal in $K$.

(1.2) [5] If $\alpha: G \rightarrow G^{\alpha}$ is a homomorphism from $G$ onto $G^{\alpha}$ and $H$ is a $\pi$-quasinormal subgroup of $G$, then $H^{a}$ is $\pi$-quasinormal in $G^{a}$.

Remark. In view of (1.1), a $(\pi-q)$-group can be defined as a group in which $\pi$-quasinormality is a transitive relation.

The following two inheritance properties of $(\pi-q)$-groups are immediate consequences of (1.1) and (1.2).

(1.3) A subnormal subgroup of a $(\pi-q)$-group is again a $(\pi-q)$-group. But a nonsubnormal subgroup of a $(\pi-q)$-group is not necessarily a $(\pi-q)$-group.

(1.4) A factor group of a $(\pi-q)$-group is a $(\pi-q)$-group.

Proposition 1.5. If $G_{1}$ and $G_{2}$ are two $(\pi-q)$-groups and $\left(\left|G_{1}\right|,\left|G_{2}\right|\right)=1$, then $G=G_{1} \times G_{2}$ is also a $(\pi-q)$-group.

Proof. Let $H$ be a subnormal subgroup of $G=G_{1} \times G_{2}$ and $G_{p}$ be a Sylow $p$-subgroup of $G$. To prove that $G$ is a $(\pi-q)$-group, we must show that $H$ and $G_{p}$ permute. Since $H$ is subnormal in $G$ and $\left(\left|G_{1}\right|,\left|G_{2}\right|\right)=1$, it is easily verified that $H=\left(H \cap G_{1}\right) \times\left(H \cap G_{2}\right)$. Clearly, we may assume without loss of generality that $G_{p} \leq G_{1}$. Since $H \cap G_{1}$ is subnormal in $G_{1}$ and $G_{1}$ is a $(\pi-q)$-group, we see that $H \cap G_{1}$ permutes with $G_{p}$. Moreover, $H \cap G_{2}$ centralizes $G_{p}$. Hence $\left(H \cap G_{1}\right) \times\left(H \cap G_{2}\right)=H$ permutes with $G_{p}$. This proves the proposition.

Remark. In this proposition, the condition that $\left(\left|G_{1}\right|,\left|G_{2}\right|\right)=1$ is necessary. The following example shows this.

Let $G_{1}=S_{3}=\left\langle x, y \mid x^{3}=y^{2}=1, y x=x^{2} y\right\rangle$ and $G_{2}=\left\langle z \mid z^{3}=1\right\rangle$. Then $\langle x z\rangle$ is subnormal in $G_{1} \times G_{2}$. But $\langle x z\rangle$ is not $\pi$-quasinormal in $G_{1} \times G_{2}$ since it does not permute with the Sylow 2-subgroup $\langle y\rangle$ of $G_{1} \times G_{2}$.

Remark. Note that a nilpotent group is always a $(\pi-q)$-group but not 
necessarily a $(q)$-group and that the class of (q)-groups defined by Zacher [8] is properly contained in the class of $(\pi-q)$-groups.

2. Solvable $(\pi-q)$-groups. In this section we will characterize the solvable $(\pi-q)$-groups. We begin with the following observation.

Lemma 2.1. Let $G$ be a $(\pi-q)$-group. If $N$ is a solvable minimal normal subgroup of $G$, then the order of $N$ is a prime.

Proof. Since $N$ is a solvable minimal normal subgroup of $G,|N|=$ $p^{n}$ for some prime $p$. Hence every subgroup of $N$, being subnormal in $G$, is $\pi$-quasinormal in $G$. Let $G_{p}$ be any Sylow $p$-subgroup of $G$. Then $N$ is a normal subgroup of $G_{p}$ and $N \cap Z\left(G_{p}\right) \neq 1$, where $Z\left(G_{p}\right)$ is the center of $G_{p}$. Let $g$ be a nonidentity element of $N \cap Z\left(G_{p}\right)$. Since $\langle g\rangle$ is $\pi$-quasinormal in $G$, it follows that $\langle g\rangle$ is subnormal in $\langle g\rangle G_{q}=G_{q}\langle g\rangle$ for all Sylow $q$-subgroups $G_{q}$ of $G$ for primes $q \neq p$. From this and the fact that $\langle g\rangle$ is a Sylow $p$-subgroup of $\langle g\rangle G_{q}$, we obtain that $\langle g\rangle \triangleleft$ $\langle g\rangle G_{q}$ for all $G_{q}, q \neq p$. But $\langle g\rangle \triangleleft G_{p}$, and hence $\langle g\rangle \triangleleft G$. Since $N$ is a minimal normal subgroup of $G$, we have $N=\langle g\rangle$ and so $|N|=p$, as desired.

Theorem 2.2. A solvable $(\pi-q)$-group $G$ is supersolvable.

Proof. We use induction on the order of $G$. Let $N$ be a minimal normal subgroup of $G$. Since $G / N$ is a solvable $(\pi-q)$-group, $G / N$ is supersolvable by induction. But $|N|$ is a prime by Lemma 2.1 and so $G$ is supersolvable.

Theorem 2.3. Let $G$ be a solvable $(\pi-q)$-group and $D(G)$ be its bypercommutator subgroup (the smallest normal subgroup of $G$ such that $G / D(G)$ is nilpotent). Then

(i) $D(G)$ is a Hall subgroup of odd order, and

(ii) every subgroup of $D(G)$ is normal in $G$. In particular, $D(G)$ is an abelian subgroup of $G$.

Remark. Note that every complement of $D(G)$ in $G$ is nilpotent.

Proof. We proceed by induction on the order of $G$. Let $p$ be the largest prime dividing $|G|$ and $G_{p}$ a Sylow $p$-subgroup of $G$. Then, since $G$ is supersolvable by Theorem 2.2 , the order of $D(G)$ is odd and $G_{p}$ is normal in $G$. We now have two cases according to whether or not $p$ divides $|D(G)|$. 
Case 1. If $p$ does not divide $|D(G)|$, then $G_{p}$ centralizes $D(G)$. By induction, $D\left(G / G_{p}\right)=D(G) G_{p} / G_{p}$ is a Hall subgroup of $G / G_{p}$ and every subgroup of $D(G) G_{p} / G_{p}$ is normal in $G / G_{p}$. This means that $D(G)$ is a Hall subgroup of $G$ and, for $H \leq D(G), H G_{p}$ is normal in $G$. But $H$ is a normal Hall (hence characteristic) subgroup of $H G_{p}$. Thus $H \triangleleft G$.

Case 2. If $p$ divides $|D(G)|$, then we will show that $G_{p} \leq D(G)$. Let $a \in G_{p}$. Since $\langle a\rangle$ is subnormal in $G,\langle a\rangle$ is $\pi$-quasinormal in $G$. Let $K$ be a $p$-complement of $G$. Then $\langle a\rangle K$ is a subgroup. Since $\langle a\rangle$ is a subnormal Sylow $p$-subgroup of $\langle a\rangle K,\langle a\rangle$ is normal in $\langle a\rangle K$, i.e., $K$ normalizes every subgroup of $G_{p}$. Hence every element of $K$ induces a power automorphism in the elementary abelian group $G_{p} / \Phi\left(G_{p}\right)$ of order prime to $p$, where $\Phi\left(G_{p}\right)$ is the Frattini subgroup of $G_{p}$. Thus, for every $k \in K$, there exists a positive integer $m(k)$ such that $a^{k} \equiv a^{m(k)}$ $\bmod \Phi\left(G_{p}\right)$ for all $a \in G_{p}$. Let $\Gamma_{n}(G)$ be the terminal member of the lower central series of $G$. Then $\Gamma_{n}(G)=D(G)$. Since $p$ divides $|D(G)|, K$ does not centralize $G_{p}$. Hence there is some $y$ in $K$ which does not centralize $G_{p}$. Now it follows from [7, Theorem 11.7] that $m(y) \not \equiv 1 \bmod p$ and so, for all $x \in G_{p}-\Phi\left(G_{p}\right)$, the commutator $\left(\left(\cdots\left(\left(x, y_{1}\right), y_{2}\right), \ldots\right), y_{n}\right)=x^{(m(y)-1)^{n}}$ $\not 1 \bmod \Phi\left(G_{p}\right)$, where $y_{1}=y_{2}=\cdots=y_{n}=y$. This says that if $A / \Phi\left(G_{p}\right) \leq$ $G_{p} / \Phi\left(G_{p}\right)$ and $\left|A / \Phi\left(G_{p}\right)\right|=p$, then $\Gamma_{n}(G)$ contains an element $g$ such that $g \in G_{p}-\Phi\left(G_{p}\right)$ and $g \Phi\left(G_{p}\right)$ generates $A / \Phi\left(G_{p}\right)$. The Burnside basis theorem yields that $G_{p} \leq \Gamma_{n}(G)=D(G)$. By induction, $D\left(G / G_{p}\right)=D(G) G_{p} / G_{p}=$ $D(G) / G_{p}$ is a Hall subgroup of $G / G_{p}$, which implies that $D(G)$ is a Hall subgroup of $G$.

Next we prove that every subgroup of $D(G)$ is normal in $G$. Clearly, we need only show that every cyclic subgroup of $D(G)$ is normal in $G$. Let $\langle c\rangle$ be any cyclic subgroup of $D(G)$. Then $c=u v=v u$ for some $p$-element $u$ and $p^{\prime}$-element $v$ and $\langle c\rangle=\langle u\rangle \times\langle v\rangle$. Since $G$ is supersolvable, $G^{\prime}$ is nilpotent. But $D(G) \leq G^{\prime}$ and so $D(G)$ is nilpotent. Hence all subgroups of $D(G)$, in particular $\langle u\rangle$ and $\langle v\rangle$, are $\pi$-quasinormal in $G$. It now follows that $\langle u\rangle \triangleleft\langle u\rangle G_{q}=G_{q}\langle u\rangle$ for all Sylow $q$-subgroups $G_{q}$ and primes $q \neq p$. Therefore, $\langle u\rangle \triangleleft G[p]$, the normal subgroup of $G$ generated by all $p^{\prime}$-elements of $G$. But $D(G) \leq G[p]$ and $G_{p} \leq D(G)$. Hence $G[p]=G$, and so $\langle u\rangle \triangleleft G$. Since $\langle v\rangle G_{p} / G_{p}$ is a subgroup of $D(G) / G_{p}=$ $D\left(G / G_{p}\right)$, we have, by induction, that $\langle v\rangle G_{p} / G_{p}$ is normal in $G / G_{p}$. Thus $\langle v\rangle G_{p} \triangleleft G$. But $\langle v\rangle G_{p}$ is nilpotent. Herice $\langle v\rangle$ is characteristic in $\langle v\rangle G_{p}$ and so $\langle v\rangle \triangleleft G$. Therefore, $\langle c\rangle \triangleleft G$ and this takes care of Case 2 . 
Finally, since $D(G)$ is Hamiltonian of odd order, it follows that $D(G)$ is abelian. Hence the proof of the theorem is complete.

The next theorem gives sufficient conditions for a group $G$ to be a $(\pi-q)$-group. Here we do not require that $G$ be solvable.

Theorem 2.4. Let the group $G$ have a normal Hall subgroup $N$ such that:

(i) $G / N$ is a $(\pi-q)$-group, and

(ii) every subnormal subgroup of $N$ is normal in $G$. Then $G$ is a $(\pi-q)$-group.

Proof. Let $H$ be a subnormal subgroup of $G$. Then we must show that $H$ is $\pi$-quasinormal in $G$. Let $N \cap H \neq 1$. Since $N \cap H$ is subnormal in $N, N \cap H$ is normal in $G$ by (ii). Now consider $G / N \cap H$. By induction, $H / N \cap H$ is $\pi$-quasinormal in $G / N \cap H$. From this it follows that $H$ is $\pi$-quasinormal in $G$.

Next suppose that $N \cap H=1$. By the improved version of the SchurZassenhaus theorem (after the well-known theorem of Feit and Thompson), $G$ splits over $N$ and all complements of $N$ are conjugate. Let $M$ be any complement of $N$ in $G$. Then $M$, being isomorphic to $G / N$, is a $(\pi-q)-$ group. Since $H$ is subnormal in $G$ and $(|N|,|M|)=1$, it is easily checked that $H=(H \cap M)(H \cap N)$. But $H \cap N=1$ and so $H=H \cap M$. This means that $H \leq M$. Hence every complement of $N$ is a $(\pi-q)$-group and contains $H$. Note that $(|H|,|N|)=1$.

Now consider the subgroup $H N$. Since $H$ is subnormal in $H N$ and $(|H|,|N|)=1$, it follows that $H$ is characteristic in $H N$. Hence $H$ permutes with every Sylow subgroup of $N$. Let $p$ be a prime divisor of the order of $G$ and $G_{p}$ be a Sylow $p$-subgroup of $G$. If $p$ divides the order of $N$, then $G_{p} \leq N$ and so $H G_{p}=G_{p} H$. On the other hand, if $p$ does not divide $|N|$, then there exists a complement $L$ of $N$ in $G$ such that $G_{p} \leq$ $L$. Since $H$ is a subnormal subgroup of $L$ and $L$ is a $(\pi-q)$-group, the subgroups $H$ and $G_{p}$ permute. Hence $H$ is $\pi$-quasinormal in $G$. This proves the theorem.

From this we obtain the following result and see that conditions (i) and (ii) of Theorem 2.3 are not only necessary but are also sufficient.

Theorem 2.5. Let $G$ have a normal Hall subgroup $N$ such that:

(i) $G / N$ is a solvable $(\pi-q)$-group, and

(ii) $N$ is solvable and all its subnormal subgroups are normal in $G$. Then $G$ is a solvable $(\pi-q)$-group. 
Proof. Since $G / N$ and $N$ are solvable, $G$ is solvable. The rest follows from Theorem 2.4.

Remark. Condition (i) of Theorem 2.5 is automatically satisfied if the factor group is nilpotent.

Corollary 2.6. Let $G$ be a solvab!e $(\pi-q)$-group. Then its subgroups are again solvable $(\pi-q)$-groups.

Proof. Let $K$ be a subgroup of $G$ and consider $K \cap D(G)$. It follows from Theorem 2.3 that $K \cap D(G)$ is a normal Hall subgroup of $K$ and its subnormal subgroups are normal in $K$. Also, $D(G) K / D(G) \cong K / K \cap D(G)$ and so $K / K \cap D(G)$ is nilpotent. Now $K$ is a solvable $(\pi-q)$-group by Theorem 2.5.

3. $(\pi-q)$-groups with special Sylow subgroups. In this section we show when a $(\pi-q)$-group, not necessarily solvable, is a $(q)$-group or a $(t)$-group. We need the following definition.

Definition. A group $G$ is called quasi-Hamiltonian if all of its subgroups are quasinormal in $G$.

Iwasawa [4] has shown the existence of quasi-Hamiltonian p-groups that are not Hamiltonian. This suggests the next theorem.

Theorem 3.1. Let $G$ be a $(\pi-q)$-group. If all Sylow subgroups of $G$ are quasi-Hamiltonian, then $G$ is a (q)-group.

Proof. Let $K$ be a subnormal subgroup of $G$. Then we must show that $K$ is quasinormal in $G$. Since the factor groups of $G$ satisfy the conditions of the theorem, it is sufficient to consider the case when the core of $K$ in $G$ (the largest normal subgroup of $G$ contained in $K$ ) is $\langle 1\rangle$. Note that $K$ is certainly $\pi$-quasinormal in $G$. By Deskins [1], $K$ is nilpotent. Hence every subgroup of $K$ is $\pi$-quasinormal in $G$. Let $p$ be a prime divisor of $|K|$. Then the Sylow $p$-subgroup $K_{p}$ of $K$ is $\pi$-quasinormal in $G$. Therefore, $K_{p}$ is normalized by every $p^{\prime}$-element of $G$ and it is contained in every Sylow $p$-subgroup of $G$. But Sylow subgroups of $G$ are quasi-Hamiltonian. Hence $K_{p}$ permutes with every $p$-subgroup of $G$. Now let $g$ be any element of $G$. Then $\langle g\rangle=\langle u\rangle \times\langle v\rangle$ for some $p$-element $u$ and $p^{\prime}$-element $v$, and so $\langle g\rangle$ and $K_{p}$ permute. Since $K$ is the direct product of its Sylow subgroups, it follows that $K$ and $\langle g\rangle$ permute, which implies that $K$ is quasinormal in $G$. This completes the proof.

Theorem 3.2. Let $G$ be a $(\pi-q)$-group. If all Sylow subgroups of $G$ are Hamiltonian, then $G$ is a $(t)$-group. 
Proof. Let $K$ be subnormal in $G$. Then, as above, $K_{p}$ is normalized by every $p^{\prime}$-element of $G$ and, since Sylow subgroups of $G$ are Hamiltonian, $K_{p}$ is a normal subgroup of every Sylow $p$-subgroup of $G$. Hence $K_{p} \triangleleft G$, and so $K \triangleleft G$.

\section{REFERENCES}

1. W. E. Deskins, On quasinormal subgroups of finite groups, Math. Z. 82 (1963), 125-132. MR 27 \#3699.

2. W. Gaschütz, Gruppen in denen das Normalteilersein transitiv ist, J. Reine Angew. Math. 198 (1957), 87-92. MR 19, 940.

3. B. Huppert, Endliche Gruppen. I, Die Grundlehren der math. Wissenschaften, Band 134, Springer-Verlag, Berlin and New York, 1967. MR 37 \#302.

4. K. Iwasawa, Über die endlichen Gruppen und die Verbände ihrer Untergruppen, J. Fac. Sci. Imp. Univ. Tokyo Sect. I 4 (1941), 171-199. MR 3, 193.

5. O. H. Kegel, Sylow-Gruppen und Subnormalteiler endlicher Gruppen, Math. Z. 78 (1962), 205-221. MR 26 \#5042.

6. O. Ore, Contributions to the theory of groups of finite order, Duke Math. J. 5 (1939), 431-460.

7. D. Passman, Permutation groups, Benjamin, New York, 1968. MR 38 \#5908.

8. G. Zacher, I gruppi risolubili finiti in cui $i$ sottogruppi di composizione coincidono con i sottogruppi quasi-normali, Atti Accad. Naz. Lincei Rend. Cl. Sci. Fis. Mat. Natur. (8) 37 (1964), 150-154. MR 30 \#833.

DEP ARTMENT OF MATHEMATICS, FERRIS STATE COLLEGE, BIG RAPIDS, MICHIGAN 49307 